\title{
MOBILE PHONE USER EXPOSURE ASSESSMENT TO UMTS AND LTE SIGNALS AT MOBILE DATA TURN ON BY APPLYING AN ORIGINAL METHOD
}

\author{
Annamaria SÂRBU, Paul BECHET, Simona MICLĂUŞ \\ "Nicolae Bălcescu" Land Forces Academy, Sibiu, Romania, \\ paljanosanna@yahoo.com, pbechet@gmail.com,simo.miclaus@gmail.com
}

\begin{abstract}
In order to provide assessment of mobile phone user exposure to electromagnetic fields emitted in the close vicinity of a mobile device we have measured real life UMTS and LTE signals at mobile data turn on. The paper presents some preliminary results of mobile phone user exposure assessment at mobile data turn by making use of a non-standardized procedure that enables the calculus of the radiated signal energy and duty cycle. Our results sustain that higher user exposure is expected within the first minute after mobile data is switched on as higher duty cycles and more than a third of the total radiated energy is found in this time period, as compared to the total signal monitoring time. Authors expect increased exposure if other application services are launched within this time frame. Moreover, special attention should be paid to user exposure assessment in the case of active call services with mobile data turned on. Because SAR is currently the sole standardized metrics in dosimetry, the authors propose the use of the measured signal radiated energy as well as the calculated duty cycle as complementary indicators of user exposure to electromagnetic fields in order to provide a more comprehensive view on mobile phone radiation exposure.
\end{abstract}

Keywords: exposure assessment, radiated power, mobile data turn on, UMTS, LTE

\section{Introduction}

The Universal Mobile Telecommunications System (UMTS) is a $3^{\text {rd }}$ Generation mobile cellular system (3G) for networks based on the Global System for Mobile Communications (GSM) standard. It is currently the most spread mobile phone communication technology for both voice and data communication services. Application services include wide-area Voice over Internet Protocol (VoIP), video calls, mobile Internet access, file transfer and streaming, all in a mobile environment. In order to meet client expectations and provide operators greater spectral efficiency as compared to $2 \mathrm{G}$ technologies, UMTS successfully implements wideband code division multiple access (WCDMA) radioaccess technology (RAT).

Mobile communication based on UMTS is already a great success. But even so, with new available RATs the end users continuously expect increased quality of serviced for both voice and data application services. In order to meet client expectations and provide high data capacities available at affordable prices operators have implemented Long Term Evolution (LTE). Based on the GSM and UMTS network technologies, LTE increases user capacity and data rate by implementing a different radio interface togetherwith other core network improvements [1]. LTE uses orthogonal frequency-division multiple access (OFDMA) modulation for the downlink and single-carrier frequency-division multiple access (SC-FDMA) for the uplink to conserve power.

For mobile telecommunication radiofrequency electromagnetic fields international safety guidelines have set 
basic restrictions in terms of Specific Absorption Rate (SAR). SAR knowledge is demanded for public information by all User Equipment (UE) producers, and its maximum value is mentioned in the technical book of each UE. Compliance assessment of UE, is performed for maximum available output power levels. However, in real operation, output power levels of a UE are generally much less than the maximum possible output power as a consequence of traffic variation, different power control mechanisms used, discontinuous transmission and soft handover [2,3]. Because, in most of the cases the operator in situated in the near field of the UE, a detailed analysis of the near field radiated power of the UE under various operating conditions and running different commonly available application services is required for an accurate realistic assessment of radiofrequency (RF) electromagnetic fields (EMF) exposure.

The use of multiband mobile devices is associated with large variations in human exposure as SAR values depend on the electromagnetic field frequency and on the actual transmit power of the device $[4,5]$. According to [5], SAR due to uplink traffic from the UE to the base station is a function of the UE's output power, the RAT duty cycle and the reference SAR (for $1 \mathrm{~W}$ of transmitted power) due to the mobile device operating at RAT.

Most of the studies on power level distributions of the uplink communication channel were found for UMTS communication technologies [6-8].

Only a few studies have been conducted to assess LTE UE output power distributions under realistic exposure scenarios $[9,10]$. Moreover, authors found no studies of comparative UMTS and LTE analysis of UE power level distributions in everyday exposure situations. In [9] authors present a series of uplink power measurements without targeting human exposure issues but rather for the purpose of deriving a power model of a commercial LTE network and comparing between $3 \mathrm{G}$ and LTE network power efficiency. Authors in [10] present result about power level distributions of $4 \mathrm{G}$ user equipment $\mathrm{UE}$ using data applications based on a very large number of samples collected over seven days in a LTE operating network. Their findings show that the mean output powers in all the environments was less than $1 \%$ of the maximum available output power and demonstrate once again that knowledge on realistic power levels is of crucial importance for accurate assessments of the radio frequency electromagnetic field exposure from mobile communication equipment.

Continuing some previous work of ours $[11,12]$, in this study we present results of an exposure assessment at mobile data turn on without the launch of any application services in both UMTS and LTE networks by making use of an original method based on near field radiated power measurements. The proposed approach enables the calculation of the radiated energy of the signal and duty cycles, parameters that can be used as complementary tools for a realistic assessment of human exposure to electromagnetic fields radiation.

\section{Materials and methods}

The proposed method is based on a measurement system composed of a spectrum analyzer connected to a miniature electric field probe. The electric field probe is to be placed in the immediate vicinity of the tested UE, and its position has to be kept constant during all measurement situations. Because we are interested in measuring the near field radiated power the channel power measurement operating should be implemented within the spectrum analyzer. In addition, it is of crucial importance to determine the optimal spectrum analyzer settings based on the measured signal characteristics.

The procedure's originality is based on measuring the UE's near field radiated power over time, which enables the 
calculation of the near field radiated energy and duty cycle. Moreover, the proposed approach enables statistical analysis that can be further used to derive worst case exposure scenarios as well as comparisons between different usage situations. In order to measure channel power over time a specially adapted Phyton code can be used for remote control of the spectrum analyzer and data collection.

The practical implementation of the proposed procedure is fully described in [12] where it was used for comparative assessment of mobile phone user exposure to UMTS and LTE signals for various data and voice application services. The materials and methods section in [12] provides a detailed discussion regarding the measurement system used as well as the optimal settings used for the spectrum analyzer. Summarizing, the main signal analyzer settings used for both generation communication technologies are presented in table 1.

Table 1 Signal analyzer optimal settings

\begin{tabular}{|l|c|c|}
\hline \multirow{2}{*}{$\begin{array}{l}\text { Signal analyzer } \\
\text { parameter }\end{array}$} & \multicolumn{2}{|c|}{ Network type } \\
\cline { 2 - 3 } & UMTS & LTE \\
\hline RBW & $500 \mathrm{kHz}$ & $1 \mathrm{MHz}$ \\
\hline VBW & $30 \mathrm{KHz}$ & $10 \mathrm{MHz}$ \\
\hline $\begin{array}{l}\text { Minimum simbol } \\
\text { duration }\end{array}$ & $0,667 \mathrm{~ms}$ & $66,7 \mu \mathrm{s}$ \\
\hline SWT & $0,667 \mathrm{~ms}$ & $66,7 \mu \mathrm{s}$ \\
\hline SPAN & $8 \mathrm{MHZ}$ & $25 \mathrm{MHz}$ \\
\hline $\begin{array}{l}\text { Number of sweep } \\
\text { points }\end{array}$ & 1500 & 6000 \\
\hline Number of sweeps & 1500 & 6000 \\
\hline $\begin{array}{l}\text { Measurement } \\
\text { period }\end{array}$ & $1 \mathrm{~s}$ & $0,4 \mathrm{~s}$ \\
\hline \multicolumn{2}{|c|}{ Source: [12] } \\
\hline
\end{tabular}

In this paper user exposure was investigated for a sole exposure scenario consisting of the UE mobile data switch on without the launch of any application service.

The dynamics of the radiated power over time enabled the calculus of the total radiated energy (W) and the duty cycle (DC) for each of the considered scenarios. The DC is defined based on eq. (1) and represents the fraction of the "active time" (when there is significant radio traffic on the channel) as compared to the total monitoring time of the channel.

$$
D C=\frac{\tau}{T} * 10
$$

where, DC is the calculated duty factor, $\tau$ is the time period for which the signal power exceeds a reference power (reference normalized power $=0.0025$ ) and $\mathrm{T}$ is the total period for which the signal was measured.

Because of the different measurement periods (see table 1) used for UMTS and LTE, greater uncertainty is expected for UMTS duty cycle calculations. Practically, any emission within 1second measurement period (UMTS) was considered active time for the entire second due to the use of max hold trace. Similarly, LTE signal was considered active if any emission was measured within $0.4 \mathrm{~s}$.

The radiated energy was obtained by integrating power over time - eq.2.

$$
W=\int_{T} P(t) d t
$$

where, $\mathrm{W}$ is the radiated signal energy during period $\mathrm{T}$ and $\mathrm{P}(\mathrm{t})$ is the measured channel power at moment $t$.

Due to the fact that only the electric field strength is measured, and that in the near field it is needed to also measure the magnetic field contribution to be able to express power and energy, the discussion below is not completely addressed. However, taking into account that human tissues are non-magnetic generally, this simplification may be acceptable.

\section{Results and discussion}

Measured power levels at mobile data turn on without launching any application were below measured power levels for any other application services considered. Cyclic increase in power level was observed without being able to define an occurrence mechanism. The calculated DC and $\mathrm{W}$ for the first minute of activity and for the entire 6 minutes duration are box-plotted in figure 1. 


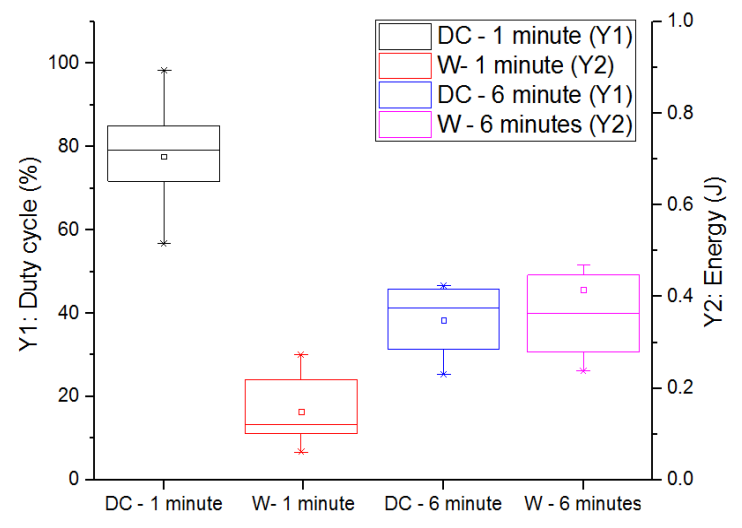

Figure 1 Duty cycle and radiated energy at mobile data turn on, for the first minute and overall 6 minutes in UMTS technology

Within the first minute after mobile data was turned on we have measured average DC of $77.6 \%$ with extreme values reaching $98.3 \%$. Overall average DC was calculated to be $20.7 \%$. We have calculated the total radiated energy of the measured signal for one minute of exposure as well as for 6 minutes exposure. We have found that $37 \%$ of the total signal energy is radiated within the first minute of exposure. Average calculated energy for 6 minutes monitoring was $0.42 \pm 0.23 \mathrm{~J}$, which indicates large data variances. Our observations sustain that at mobile data switch, without the launch of any application, highest exposure occurs within the first minute of exposure when also higher duty cycles are measured and more than a third of the total energy is being stored.

Figure 2 presents LTE radiated power dynamics at mobile data switch on, without launching any application service. Each point in the scatter plot represents the averaged value of 10 different measurements.

Within the first minute after mobile data turn on, measured radiated power constantly decrease, reaching about a half of the initial value. A short decrease followed by a rapid increase was observed after the first 30 seconds of activity. Further on, we have observed cyclic increases in radiated power, at estimated period ranges of 20-60 seconds. We were able to deduct an empiric law for the decrease rate of radiated power with time, with a calculated correlation coefficient $\mathrm{R}^{2}$ of 0.59 .

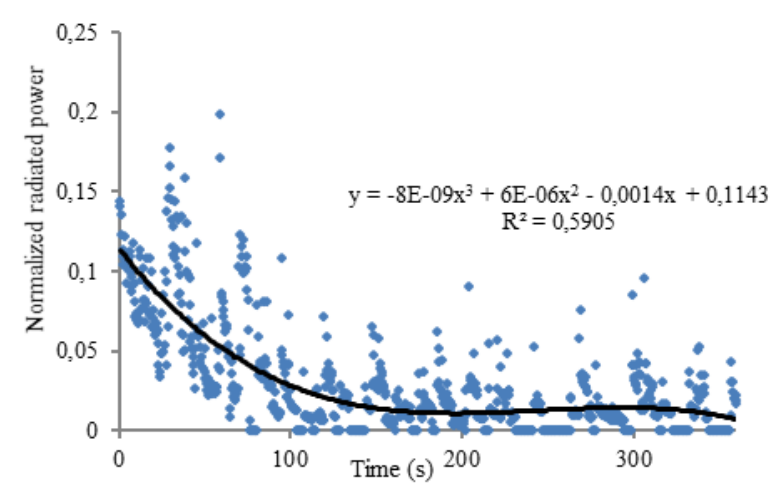

Figure2 LTE radiated power evolution in time at mobile data switch on

Based on these observations, we can state that the user is expected to be more exposed during the first minute after mobile data is switched on. If other application services are launched within this time frame additional radiated power is expected to be added to the measured values. Special attention should be paid to user exposure assessment in the case of active call services with mobile data turned on.

In terms of calculated DC and radiated energy at mobile data turn on, figure 3 presents the main differences between LTE and UMTS communication standards.

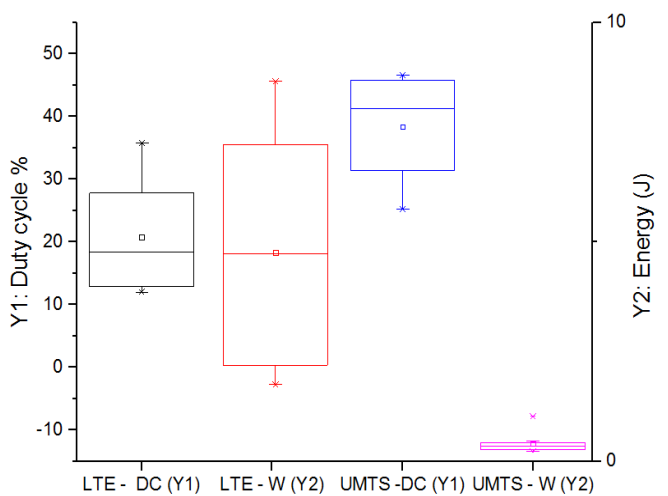

Figure 3 LTE and UMTS comparative DC-6 minutes and radiated energy at mobile data turn on

The average 6 minutes DC calculated for LTE was of $19.54 \%$ while the average first minute DC was $61.63 \%$ with extreme values 
reaching 93.3\%. Compared to UMTS DC at mobile data turn on (see fig.1) higher DCs were calculated for UMTS than for LTE. Previous observations of higher radiated energies for LTE compared to UMTS were also found for previously investigated exposure scenarios. [12]

\section{Conclusions}

Generally, differences due to exposure mechanisms are hard to be quantified because of the complex interactions between the human body and EMFs, but applying this procedure one may rank among exposure associated with different everyday scenarios.

The procedure applied here enables the calculation of the total signal radiated energy and duty cycle, which can be used as complementary measures of one's exposure. Moreover, by applying this procedure exposure may be investigated by means of a swept spectrum analyzer which is more widely available than a real time spectrum analyzer.

By applying the procedures we have found higher user exposure is expected within the first minute after mobile data is switched on as higher duty cycles and more than a third of the total radiated energy is found in this time period, as compared to the total signal monitoring time. However, the highest energies calculated for LTE network as compared to UMTS network sustain our previous conclusion that highest exposure is expected for the operator under LTE rather than under UMTS communication standard, during the use of similar application services.

The results reported here represent a starting point for future research which includes procedure refinements as well as measurements of the near field radiated power and duty cycles under various propagation conditions and real life exposure scenarios.

\section{References}

[1] 3rd Generation Partnership Project (3GPP), TS 36.101 version 10.3.0 Release 10, LTE; Evolved Universal Terrestrial Radio Access (E-UTRA); User Equipment (UE) radio transmission and reception

[2] T. Persson, C. Törnevik, L.-E. Larsson, and J. Lovén, "Output power distributions of terminals in a $3 \mathrm{G}$ mobile communication network," Bioelectromagnetics, vol. 33, no. 4, pp. 320-325, May 2012.

[3] P. Joshi, "Assessment of realistic output power levels for LTE devices," M.S. thesis, Dept. Elect. Inf. Technol., Lund Univ., Lund, Sweden, 2012.

[4] ICNIRP, ICNIRP Guidelines for limiting exposure to time-varying electric, magnetic and electromagnetic fields up to $300 \mathrm{GHz}$, published in Health Physics, vol. 74 (4), pp. 494-522, 1998

[5] Plets D. et. all, Joint minimization of uplink and downlink whole-body exposure dose in indoor wireless networks, BioMed Research International, Volume 2015, http://dx.doi.org/10.1155/2015/943415

[6] Jovanovic D., Bragard G., Picard D. and Chauvin S., Mobile telephones: A comparison of radiated power between $3 \mathrm{G}$ VoIP calls and $3 \mathrm{G}$ VoCS calls, Journal of Exposure Science and Environmental Epidemiology (2015) 25, pp. 80-83

[7] Joshi P. et.all, Power level distribution of radio base station equipment and user device in a 3 Gmobile communication network in India and the impact on assessment of realistic EMF exposure, IEEE Access , 2015, doi 10.1109/Access.2015.2453056

[8] Persson T., Tornevik C., Larsson L.E., Loven J., Output power distributions of terminals in a $3 \mathrm{G}$ mobile communication network, Bioelectromagnetics, Vol.33, 2012, pp. $320-325$ 
[9] Huang J. et.all, A close examination of performance and power characteristics of 4G LTE networks, MobiSys '12 Proceedings of the 10th international conference on Mobile systems, applications, and services, 2012, pp. 225-238

[10] Paramananda Joshi, Davide Colombi, Björn Thors, Lars-Eric Larsson, and Christer Törnevik, Output Power Levels of 4G User Equipment and Implications on Realistic RF EMF Exposure Assessments,

[11] Miclaus S., Bechet P., Comparative characterization of the electromagnetic near field radiated by mobile phones in GSM and UMTS communication technologies, International Conference and Exposition on Electrical and Power Engineering (EPE), 2014, pp.471-475, doi: 10.1109/ICEPE.2014.6969952

[12] Paljanos A., Miclăuş S., Bechet P., Munteanu C., Assessment of mobile phone user exposure to UMTS and LTE: comparative near field radiated power evaluation for various types of application services, Journal of Electromagnetic Waves and Applications, doi: 10.1080/09205071.2016.1167634, 2016 